\title{
CONCEPTS
}

\section{Retrieval of Additional Epinephrine from Adrenaclick-Style Epinephrine Autoinjectors}

\author{
Arun Ganti, MD, MPHS ${ }^{1}$; Rebecca Pilkerton, $\mathrm{MD}^{2}$; Erika Falsgraf, $\mathrm{MD}^{1}$; Elizabeth Vanwert, PharmD, BCPS ${ }^{3}$; \\ Nathan Brouwer, $\mathrm{MD}^{4}$; Alexander Beyer, $\mathrm{MD}, \mathrm{MPHS}^{4}$; Brendan Byrne, $\mathrm{MD}^{4}$ \\ ${ }^{1}$ Department of Emergency Medicine, UCSF Fresno, Fresno, CA; ${ }^{2}$ Department of Emergency Medicine, University of Utah, Salt Lake City, UT; ${ }^{3}$ Depart- \\ ment of Pharmacy, Michigan Medicine, Ann Arbor, MI; ${ }^{4}$ Department of Emergency Medicine, Michigan Medicine, Ann Arbor, MI
}

\begin{abstract}
Anaphylaxis is a life-threatening allergic reaction involving multiple organ systems that can result in significant morbidity and mortality if left untreated. Epinephrine is the mainstay of treatment. Most episodes of anaphylaxis resolve after a single dose of epinephrine, but biphasic and protracted courses of anaphylaxis are well described. The need for additional doses of epinephrine poses a significant challenge in the wilderness setting, because patients and providers may only carry a single autoinjector. Prior work has demonstrated successful disassembly of various brands of epinephrine autoinjectors to retrieve additional drug product for repeat dosing. We describe 2 techniques to retrieve additional doses of epinephrine from Adrenaclick-style epinephrine autoinjectors. The techniques described are off-label and are not approved by the manufacturer or the Food and Drug Administration. Wilderness providers should familiarize themselves with techniques for retrieval of additional epinephrine from various autoinjectors in light of significant differences in product design.
\end{abstract}

Keywords: anaphylaxis, wilderness medicine, allergic reaction

\section{Introduction}

Anaphylaxis is a life-threatening allergic reaction involving multiple organ systems that can result in airway compromise, hypotension, and death. If untreated, anaphylaxis results in significant morbidity and mortality, and the potential causes in an austere setting are myriad. ${ }^{1,2}$

Epinephrine is the mainstay of treatment, primarily delivered through easy-to-use autoinjectors designed for single use. Although most episodes of anaphylaxis resolve after a single dose of epinephrine, biphasic and protracted courses of anaphylaxis are well described. ${ }^{3}$ Biphasic cases occur approximately $20 \%$ of the time, the majority within $8 \mathrm{~h} .{ }^{4}$ To address the need for redosing, epinephrine autoinjectors are intentionally sold as 2-packs. The need for additional doses of epinephrine poses a significant challenge in the wilderness setting, because patients and providers may

Corresponding author: Arun Ganti, MD, MPHS, FAWM, Department of Emergency Medicine, UCSF Fresno, 155 N. Fresno Street, Suite 201, Fresno, CA 93701; e-mail: aganti@ fresno.ucsf.edu.

Submitted for publication January 2020.

Accepted for publication September 2020. carry only a single autoinjector owing to weight or space concerns, financial constraints, or improper planning. If additional doses are needed but are unavailable, used autoinjectors can be disassembled to retrieve extra doses of epinephrine contained inside the ampoule.

The past decade has seen the introduction, recall, discontinuation, and redesign of several epinephrine autoinjectors in the US market. Currently, 4 styles of epinephrine autoinjectors are available in the United States: Auvi-Q (Kaléo), Adrenaclick (Amneal Pharmaceuticals), Symjepi (Sandoz), and EpiPen (Mylan). Generic versions of EpiPen and Adrenaclick are also available. Prior work has demonstrated successful disassembly of EpiPen and Auvi-Q autoinjectors to retrieve additional drug product for repeat dosing. ${ }^{5,6}$ The retrieval of additional epinephrine from Adrenaclick-style epinephrine autoinjectors (ASEAs) using pliers was described using a similarly designed product, the Twinject. ${ }^{5,6}$ Although techniques can be adapted from one style of autoinjector to another, the construction of different brands varies enough to require detailed instructions specific to each type of autoinjector. We describe in detail 2 techniques, 1 using pliers and 1 using a knife, to retrieve additional doses of epinephrine from ASEAs. To 
our knowledge, the knife technique has not been previously described and offers an alternative when pliers are not available. The techniques described are off-label and are not approved by the manufacturer or the Food and Drug Administration.

\section{Methods}

\section{ADMINISTERING THE FIRST DOSE}

We recommend following the manufacturer's instructions to deliver the first dose of epinephrine, with 1 caveat: We do not recommend delivery through thick clothing because this may damage the needle and prevent its reuse.

\section{SAFETY CONCERNS}

Unlike the EpiPen, ASEAs do not protect the needle after firing (Figure 1). Be mindful of the exposed needle to avoid damaging it during disassembly and to avoid a needlestick injury. It is also important to recognize that this needle has been used; this technique is not intended for treatment of multiple patients.

\section{REMOVING THE SYRINGE FROM THE CARTRIDGE}

\section{Technique \#1-Pliers}

Step 1: Using pliers, grab hold of the red cap just below the needle and twist off this segment (Figure 2). This will require a significant amount of force.

Step 2: The epinephrine ampoule can then be removed (Figure 3).

\section{Technique \#2-Knife}

Step 1: Remove the sticker on the outer plastic housing to expose the underlying components.

Step 2: Identify the epinephrine ampoule and the spring at the opposite end from the needle. Use a knife to cut the outer housing where they meet. Do not attempt to cut at the red cap or at the groove between the outer and inner housings because doing so risks damaging the syringe during this process (Figure 4). The plastic housings for these autoinjectors are made of harder material than that used

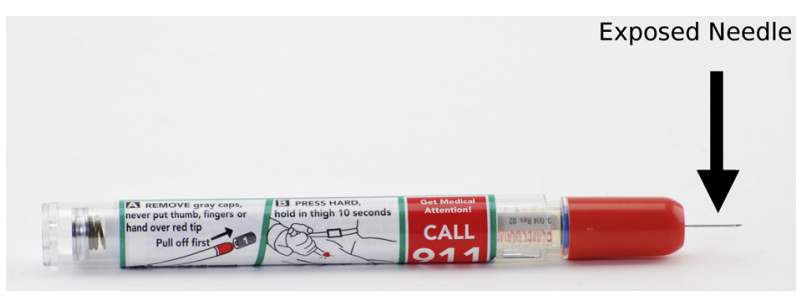

Figure 1. Used autoinjector with exposed needle.

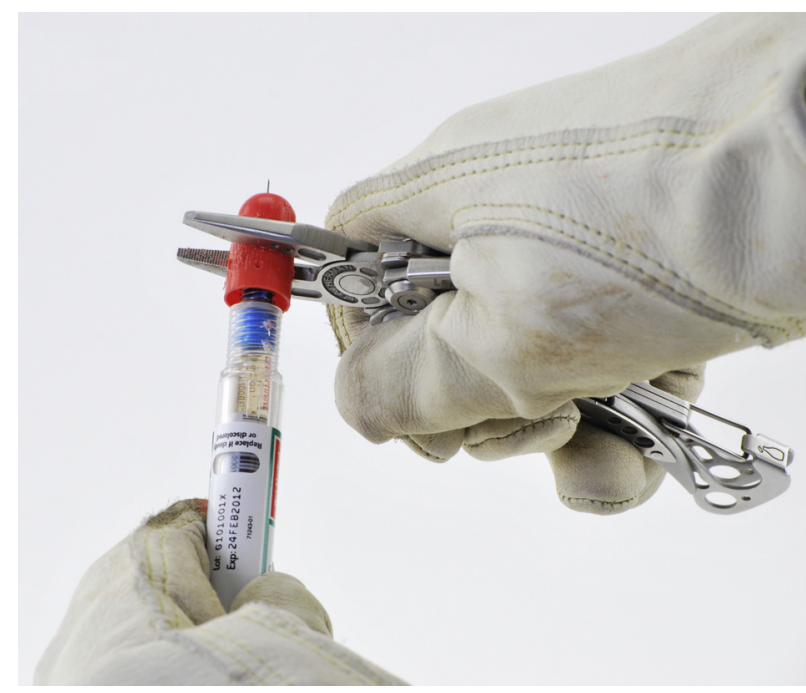

Figure 2. Grab red cap with pliers and twist off assembly.

for the EpiPen. In our experience, it is easier to use a sawing technique with a serrated blade rather than an incision with a straight edge to cut through the plastic (Figure 5). With either blade type, one must pay close attention to blade safety; the hard plastic creates the potential for blade slippage, resulting in self-injury. Once the outer housing has been fully cut, remove it to expose the inner housing (Figure 6).

Step 3: Cut the inner housing and retrieve the epinephrine ampoule (Figure 7).

\section{ADMINISTERING ADDITIONAL DOSES}

Before administering an additional dose, it is necessary to draw air into the ampoule. This is required because the specialized plunger only allows for the administration of the prespecified single dose, owing to restricted travel within the ampoule. The air fills the space of the administered drug, which resets the plunger and allows one to inject additional medication. With the needle pointing up, slowly withdraw the plunger to draw the desired amount of air into the ampoule. Be careful not to completely withdraw the plunger; this poses the risk of losing the drug. The ampoule has markings to ensure accurate dosing $(0.3 \mathrm{~mL}$ for adults, $0.15 \mathrm{~mL}$ for children) and to display how much drug is available (Figure 8). To administer the second dose, insert the needle into the lateral thigh and inject.

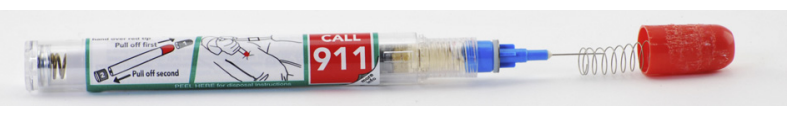

Figure 3. Autoinjector with red cap removed, allowing for retrieval of epinephrine syringe. 


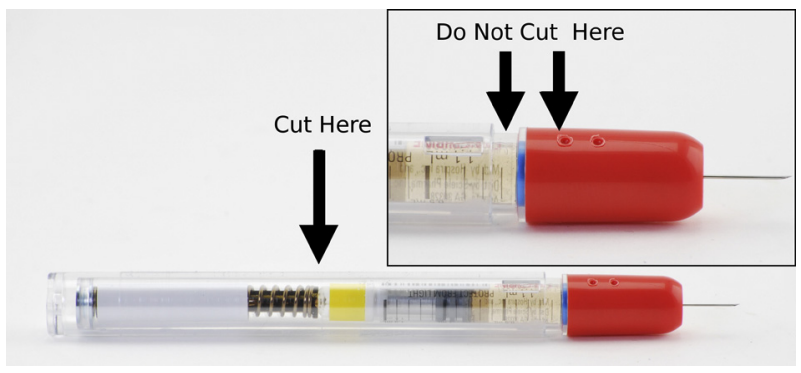

Figure 4. Remove sticker to expose plastic casing. Cut the autoinjector where the ampoule and spring meeting. Do not cut at the red cap or groove between the outer and inner housings.

The ampoule contains 3 total doses. A second additional dose can be administered by repeating these steps.

These steps are outlined in the supplementary video.

\section{Discussion}

We describe 2 techniques for retrieval of additional doses of epinephrine from ASEAs. The pliers technique has been previously described. ${ }^{6}$ The knife technique is novel and adds to the literature by providing an alternative approach if pliers are not available. The harder plastic and dual housing design of ASEAs requires a different technique than that used to disassemble an EpiPen when using a knife. EpiPen disassembly involves cutting through a single housing and removing a plastic stopper from the base of the autoinjector. ${ }^{6}$ In contrast, ASEA disassembly requires cutting through 2 separate housings. Cutting at different sites, including the red cap at the needle end or the groove between the 2 housings, will not allow easy access the ampoule and may damage the ampoule or needle in the process. Based on our experience, we find that the pliers technique is the easier of the 2 described methods.

The techniques described previously and in this publication are off-label and should only be considered as

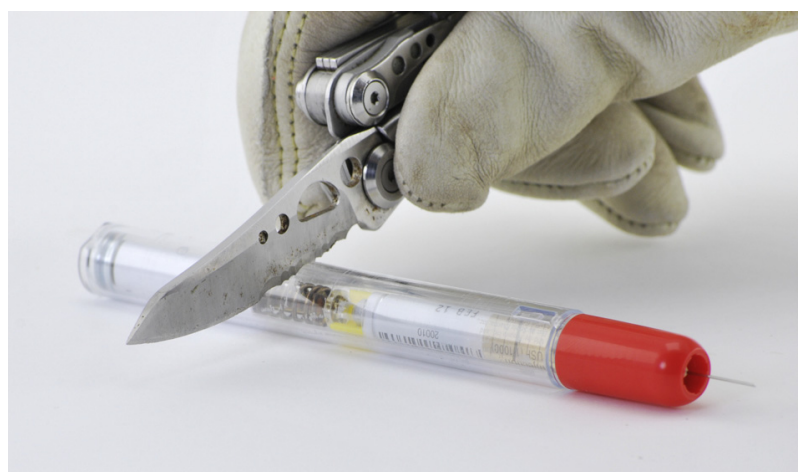

Figure 5. Cut outer housing.

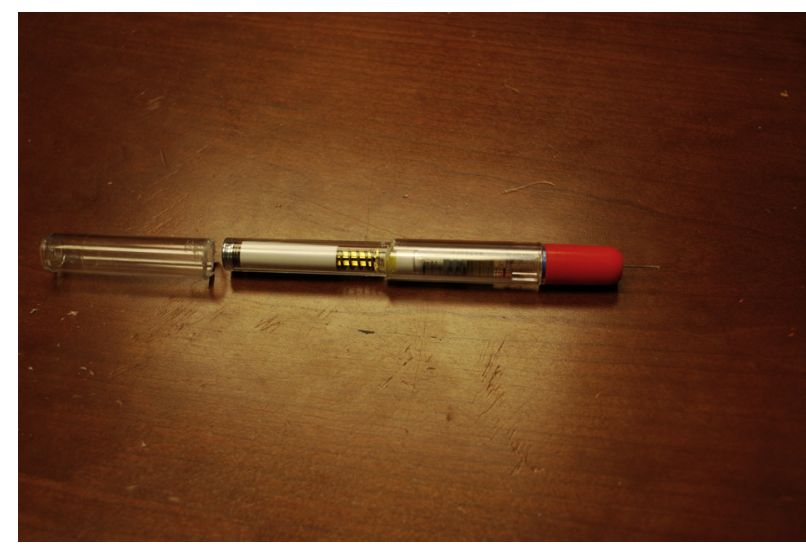

Figure 6. Outer housing removed.

temporizing measures when additional epinephrine autoinjectors are unavailable and arrival of prehospital personnel or transport to the hospital is delayed. Although these techniques describe a viable means to get additional epinephrine doses from an ASEA, the multiple steps required coupled with the exposure to blades and used needles should relegate this practice to exigent circumstances only. During disassembly, there is a risk the needle could be contaminated before reuse. The person performing the disassembly is at risk of knife or needlestick injury. In addition, administering epinephrine with the syringe requires a higher level of expertise than use of an autoinjector.

A core tenet of wilderness medicine is that proper planning and preparation are superior to emergency repurposing of the limited resources available in a wilderness environment. Proper consideration of hazards, planning for treatment, and evacuation would mitigate the need for the techniques described in this paper. We do not recommend using knowledge of these techniques to justify carrying fewer autoinjectors or to avoid the need to seek additional care after epinephrine administration. Sufficient supplies of epinephrine autoinjectors should be carried by those with known allergies traveling to remote settings and by personnel providing care in these settings. Consistent with Wilderness Medical Society guidelines, we recommend that any patient with anaphylaxis seek advanced medical care as soon as possible. ${ }^{7}$ Nevertheless, we believe that understanding the techniques described here may prove vital to mitigating the effects of life-threatening anaphylaxis in an austere environment when additional resources are not readily available.

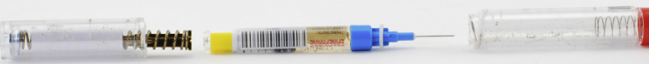

Figure 7. Cut inner housing and retrieve epinephrine syringe. 


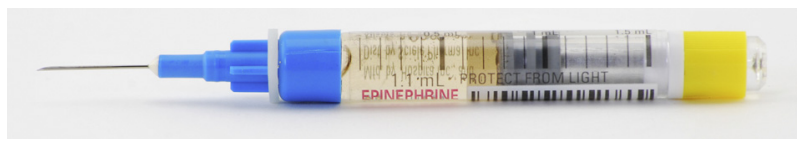

Figure 8. Syringe with epinephrine.

\section{LIMITATIONS}

Despite containing the same drug, the construction of epinephrine autoinjectors varies significantly among manufacturers. The technique to retrieve additional drug product for repeat dosing is unique for each of these designs. As such, these techniques are only valid for as long as this style of epinephrine autoinjector is available commercially. The exposed needle in this style of epinephrine autoinjector poses a risk for significant injury or body fluid exposure. These techniques are only to be used when all other options are exhausted. We do not endorse using this as a strategy to avoid carrying sufficient quantities of epinephrine or to avoid the need to seek additional care after administration of epinephrine.

\section{Conclusions}

Anaphylaxis is a life-threatening allergic reaction involving multiple organ systems that requires prompt recognition and treatment with epinephrine. The priority is administration of epinephrine followed by immediate transport to a hospital. Wilderness medical providers and outdoor enthusiasts with a history of anaphylaxis are responsible for maintaining an adequate supply of epinephrine by carrying multiple autoinjectors to account for the possibility of biphasic reactions and prolonged transport times. When additional autoinjectors are not readily available, the described techniques may prove vital to mitigate the effects of life-threatening anaphylaxis in an austere environment. Wilderness providers should familiarize themselves with techniques for retrieval of additional epinephrine from various autoinjectors in light of significant differences in product design.
Acknowledgments: Special thanks to Dr. Keerthi Karamched from the Michigan Medicine Division of Allergy and Immunology for donating expired autoinjectors and to Dr. Brad Smith from the University of Michigan Stamps School of Art \& Design for assistance with photography and videography.

Author Contributions: Study concept and design (AG, EV, NB); collection of materials (AG); literature review (AG, EF, RP, EV, BB); video editing (RP); manuscript writing and editing (all); final approval of manuscript and video (all).

Financial/Material Support: This study received no funding support. Expired autoinjectors were donated by providers from the Michigan Medicine Division of Allergy and Immunology.

Disclosures: None.

\section{Supplementary data}

Supplementary data to this article can be found online at https://doi.org/10.1016/j.wem.2020.09.004.

\section{References}

1. Tejedor Alonso MA, Moro Moro M, Múgica García MV. Epidemiology of anaphylaxis. Clin Exp Allergy. 2015;45(6): 1027-39.

2. Yu JE, Lin RY. The epidemiology of anaphylaxis. Clin Rev Allergy Immunol. 2018;54(3):366-74.

3. Pumphrey R. Anaphylaxis: can we tell who is at risk of a fatal reaction? Curr Opin Allergy Clin Immunol. 2004;4(4): 285-90.

4. Boyce JA, Assa'ad A, Burks AW, Jones SM, Sampson HA, Wood RA, et al. Guidelines for the diagnosis and management of food allergy in the United States: report of the NIAIDsponsored expert panel. J Allergy Clin Immunol. 2010;126(6 Suppl):S1-58.

5. Robinson PE, Lareau SA. Novel technique for epinephrine removal in new generation autoinjectors. Wilderness Environ Med. 2016;27(2):252-5.

6. Hawkins SC, Weil C, Baty F, Fitzpatrick D, Rowell B. Retrieval of additional epinephrine from auto-injectors. Wilderness Environ Med. 2013;24(4):434-44.

7. Gaudio FG, Lemery J, Johnson DE. Wilderness Medical Society practice guidelines for the use of epinephrine in outdoor education and wilderness settings: 2014 update. Wilderness Environ Med. 2014;25(4 Suppl):S15-8. 